\title{
Magic of Fibonacci Sequence in Prediction of Stock Behavior
}

\author{
Rajesh Kumar \\ $943 \mathrm{~A} / 28$ \\ Bharat colony, Rohtak, Haryana.
}

\begin{abstract}
Predicting the return of a financial product is a very risky task. It involves subjectivity and experts knowledge. In the development of an expert system, domain knowledge is one of the important component. For a software to be artificial intelligent, some heuristics are required, which can help in decision making. It is admitted by the technical experts of financial sectors that in predicting the support or resistance backtracking is required when prediction of support or resistance fails. In this paper, an attempt has been made to restrict the back tracking of support and resistance to a maximum of two attempts. Proposed model can be further used in machine learning to remove the subjectivity.
\end{abstract}

\section{Keywords}

Fibonacci sequence, Factor analysis, principal component analysis, RSI, MFI.

\section{INTRODUCTION}

Financial markets are constantly generating large volume of data. Analyzing these data to reveal valuable information is a challenging task. When pattern or behavior of a financial time series is understood then it provides a very good opportunity of earning money. Most financial data are random time series containing noisy data, nonlinear data and non stationary behavior thus making it difficult to model. Owing to these problems, some satisfactory solution is required as the optimal solution is not possible. A time series is a sequence of real numbers that represent values of a real variable measured at equal time intervals. For example, a time series can represent movements of stock prices or exchange rates of a security. Investors in the market want to maximize their returns by buying or selling their securities of their choice at an appropriate time, but taming it without any statistical or other evolutionary algorithm is a difficult task. Since stock market data are highly time-variant and are normally in a nonlinear pattern, predicting the future trend of a stock is a challenging problem because of the subjectivity of the emotions of the investors as emotions are very difficult to model. Prior research has demonstrated that the prediction of future returns of individual stocks can be based on the growth rates of a number of fundamental factors such as revenues, earnings per share, capital investment, debt, market share and few other financial ratios [12]. Regression models have been traditionally used to model changes in the stock markets. However, those models can predict linear patterns only [13]. Typically, the performance of a model in classification problems such as stock prediction is measured by prediction accuracy. Most neural network models that attempt to predict individual stocks only use information from the respective markets. Some studies attempt to include not only the current stock index value but also traded volumes from all indexes. In the neural network models to estimate the effect of more established markets whose values affect the performance of smaller emerging markets [14]. In prediction of stock market genetic algorithm and other evolutionary algorithms are widely used. Greatest challenges in prediction is its subjectivity, two analyst can have different interpretation of a scenario. In this paper, an attempt has been made to make the prediction objective so that machine learning can be achieved . Fibonacci series is widely used in financial market to predict the resistance and support levels, off course this method of retracement prediction has great accuracy but its drawback is that prediction of retracement is not a single values but a set of values. In this paper an attempt has been made to classify the retracement values to the maximum of cardinality two. Ex. model will predict that retracement will be either $50 \%$ or $61.8 \%$. In this model RSI and MFI are noted at highest peak and then retracement will be predicted on taking decisions on these independent variables. Rest of the paper is organized as follows

Section 2. covers recurrence relation.

Section 3 . covers factor analysis.

Section 4. covers Fibonacci sequence.

Section 5. covers indicators.

Section 6. proposed model.

Section 7. covers data analysis.

Section 8. covers conclusion.

Section 9. covers references.

\section{RECURRENCE RELATION [1]}

The fundamentals tools permutation and combination are inadequate to solve the many combinatorial problem. In such cases recurrence relation are frequently used to solve the problems. Recurrence relation are used in many counting problems and in analyzing programming problems. They occur in analysis of certain discrete time systems ,analysis of algorithms and error correcting codes. In recurrence, previous values are used to compute the current values. A recurrence relation is of the following form.

$$
\mathrm{f}(\mathrm{n})=c_{0} a_{n}+c_{1} a_{n-1}+c_{2} a_{n-2}++c_{k} a_{n-k}
$$

is called a linear recurrence relation with constant coefficients having order $\mathrm{k}$. Recurrence relation is like a recursively defined sequence but without specifying any initial values hence it can have multiple solutions. If initial values are provided than unique solution can be determined.

There are various methods of solution of a recurrence relation. In the substitution methods of solution of recurrence relation for $a_{n}$ is used repeatedly to solve a general expression for $a_{n}$ in terms of $n$ such that the expression does not contain any other 
terms of the sequence except those given by the initial condition. Generally it is preferred to have an explicit formula to compute the value of $a_{n}$ rather than conducting $n$ iterations, hence characteristics root method is preferred.

The Fibonacci numbers satisfy the recurrence relation $f_{n}=f_{n-1}$ $+\mathrm{f}_{\mathrm{n}-2}$ with initial conditions $\mathrm{f}_{0}=0$ and $\mathrm{f}_{1}=1$.

The characteristic equation is $r^{2}-r-1=0$.

Its roots are

$$
\begin{aligned}
& r 1=(1+\sqrt{5}) / 2 \\
& r 2=(1-\sqrt{5}) / 2
\end{aligned}
$$

Therefore Fibonacci numbers are given by

$$
f(n)=\alpha 1\left(\frac{1+\sqrt{5}}{2}\right)+\alpha 2\left(\frac{1-\sqrt{5}}{2}\right)
$$

Putting the initial conditions to obtain $\alpha_{1}$ and $\alpha_{2}$.

$$
\begin{aligned}
& \alpha 1=\frac{1}{\sqrt{5}} \\
& \alpha 2=\frac{-1}{\sqrt{5}}
\end{aligned}
$$

So Fibonacci sequence is obtained by the following equation.

$$
f(n)=\frac{1}{\sqrt{5}}\left(\frac{1+\sqrt{5}}{2}\right)^{\mathrm{n}}+\frac{-1}{\sqrt{5}}\left(\frac{1-\sqrt{5}}{2}\right)^{\mathrm{n}}
$$

\section{FACTOR ANALYSIS}

Factor analysis is used to identify a small number of factors that may be used to represent the relationship among the sets of interrelated variables. Following steps are required to conduct a factor analysis[2].

- calculate a correlation matrix of all variables to be used in the analysis.

- Extraction of factors.

- Rotate factors to create a more understandable factor structure.

Factor extraction method starts with a measure of total amount of variation observed in all variables under the study of factor analysis. The first step in factor analysis is for the computer to select the combination of variables whose shared correlation explains the greatest amount of the total variance as called factor1. Then for the search of second component is done, followed by third component and so on. Eigen values are designated to display the proportion of the variance accounted for each factor[2]. Generally all factors with eigen values greater than one are kept and rest are dropped. Scree plot may be used for the factor selection[2]. Rotation is needed owing to the difficulty in its interpretation. The goal of rotation is to achieve the simple structure by high factor loading on one factor and low loading on others. Rotation does not change the mathematical accuracy of the factor structure. Verimax rotation called the orthogonal rotation because the axes that are rotated remain at right angles to each other[2]. Factors that have the high loadings will have excellent face validity and appear to be measuring some constructs. Some times a variable is loaded on the wrong factor and similarly loading of a variable into two or more factors is also possible [2]. Principal component analysis is the default method of factor extraction in PSPP. The properties of principal components are based on the Eigen vectors and Eigen values of the covariance matrix[3]. Principal component is defined as $\mathrm{Z}=\mathrm{A}^{\prime} \mathrm{X}^{*}$ where $\mathrm{A}$ has columns consisting of the eigenvectors of the correlation matrix, and $\mathrm{X}^{*}$ stands for the standardized variables[3].

\section{FIBONACCI SEQUENCE [10]}

The Fibonacci sequence is the series of numbers $0,1,1,2,3$, $5,8,13,21,34 \ldots \ldots \ldots \ldots$ The next number is found by adding up the previous two numbers. Hence Fibonacci sequence can be defined by a mathematical formula $f(n)=f(n-$ 1) $+\mathrm{f}(\mathrm{n}-2)$. Fibonacci sequence successfully predict and analyze the price trend of the stock market. In golden ratio sum of the two quantities to the larger quantities is approximately $1.6180339887 . .$, similarly ratio of two successive numbers of Fibonacci sequence is approximately 1.618034. Fibonacci retracement is used in finding the support and the resistance of a financial time series. In this method major peak and trough are identified, then it is followed by dividing the vertical distance into $23.6 \%, 38.2 \%, 50 \%, 61.8 \%$ and $100 \%$. As per the Eliott wave theory, Fibonacci sequence and relationships between various waves has been observed and given below.

Wave 2 is either $50 \%$ or $61.8 \%$ retracement of Wave 1 .

- Fibonacci Relationship for wave 3 is

Wave 3 is either 1.618 times the length of wave 1 or

Wave 3 is 2.618 times the length of wave 1 or

Wave 3 is 4.23 times the length of wave 1 .

- Fibonacci Relationship for wave 4 is

wave 4 is always related to wave 3 .

wave 4 is either $23.6 \%$ retracement of wave 3 or

wave 4 is $38.2 \%$ retracement of wave 3 or

wave 4 is $50 \%$ retracement of wave 3

- Fibonacci Relationship for wave 5 and related to wave 1 indirectly.

(1) If wave 3 is more than 1.618 times the length of wave 1 . then

wave 5 is either wave 1 or

wave 5 is 1.618 times the length of wave 1 or

wave 5 is 2.618 times the length of wave 1 .

(2). Wave 5 is related to entire length from bottom of wave 1 to the top of wave 3 and If wave 3 is less than 1.618 times the length of wave 1 then wave 5 is either 1.618 times the entire length from bottom of wave 1 to the top of wave 3 . or

wave 5 is 2.618 times the entire length from bottom of wave 1 to the top of wave 3 .

In any case wave 4 is not more than $61.8 \%$ retracement of wave3. 
- Fibonacci Relationship for wave A is

wave $\mathrm{A}$ is either $23.6 \%$ retracement of wave 5 or

wave $\mathrm{A}$ is $38.2 \%$ retracement of wave 5 .

- $\quad$ Fibonacci Relationship for wave B is

wave $B$ is related to wave $A$.

wave B is either $50 \%$ retracement of wave A or

wave B is $61.8 \%$ retracement of wave A.

- $\quad$ Fibonacci Relationship for wave $\mathrm{C}$ is

.wave $\mathrm{C}$ is either 1.618 times the length of wave $\mathrm{A}$ or wave 3 is 2.618 times the length of wave A.

\section{INDICATORS}

There are various indicators to facilitate the trading decisions but none of them alone can predict the behavior of the market as the subjectivity of the market. Various indicators like POS, Chaikin, money flow oscillator, money flow indicator and relative strength indicator are frequently used in technical analysis. POS s a momentum oscillator with some trend-following characteristics. It expresses the difference between two moving averages as a percentage value that quantifies where the fast moving average is in relation to the slow moving average. The Percentage Price Oscillator is very similar to the Moving Average Convergence or Divergence (MACD), except the MACD expresses the difference of two moving averages as an absolute value. Expressing the difference as a percentage value makes it easier to compare trading instruments with difference prices[10]. Chaikin money flow oscillator is calculated from the daily readings of the accumulation and distribution Line. The basic principal behind the accumulation or distribution Line is that the degree of buying or selling pressure. The formula for Chaikin money flow is the cumulative total of the accumulation /distribution Values for 21 periods divided by the cumulative total of volume for 21 periods[4]. MFI is based on the concept of money flow. If typical price calculated by (High+ Low + Close $) / 3$ of today is higher than the previous day, it is said that typical price * volume amount of money has flown into the security as vice versa. MFI is an oscillator, whose value is calculated using the ratio of total positive money flow in the last $\mathrm{N}$ days to the negative money flow in the same duration. High values of MFI indicate overbought levels, where as low values indicate oversold levels. Positive and negative divergences between the stock and the MFI (Prices move in one direction and MFI moves in the opposite direction) can be used as buy and sell signals respectively with reversal of a trend[5]. RSI is an oscillator, whose value oscillates between $0-100$. It is calculated based on relative strength as a measure of momentum. An N-period relative strength is the ratio of gains in the price in last $\mathrm{N}$ days, divided by the losses in last $\mathrm{N}$ days. A RSI of 70 or above can indicate a stock which is overbought and due for a fall in price. When the RSI falls below 30 the stock may be oversold, whereas these marks of RSI are different in bull market and bear market [4].

\section{PROPOSED MODEL}

Stock prediction clue is taken from various indicators and annotations. Various graphs are available but prediction decision is quite subjective and is stock specific. In this model, various indicators like POS, Chaiken money flow, MFI and RSI are taken as the independent variable whereas retracement of wave is taken as the dependent variable. Factor analysis was done to extract the relevant attributes with significance. Principal component analysis was used for the factor analysis and found out that component1 was having Eigen value as 2.64 and component 2 has Eigen value .75 with a variance of 66.01 and 18.72. In this paper, a rise from trough to crust is noted and its retracement is observed, In other words it is stated that falling wave as called wave2 retracement is studied with a relation with wave 1 .

Table 1 . Communities table

\begin{tabular}{|l|l|l|}
\hline Attribute & Initial & Extraction \\
\hline CHI & 1 & .44 \\
\hline POS & 1 & .60 \\
\hline RSI & 1 & .89 \\
\hline MFI & 1 & .71 \\
\hline
\end{tabular}

Table 2. Explanation of variance

\begin{tabular}{|l|l|l|l|}
\hline Component & \multicolumn{3}{|l|}{ Initial Eigen values } \\
\hline & Total & $\begin{array}{l}\text { Percentage } \\
\text { of variance }\end{array}$ & $\begin{array}{l}\text { Cumulative } \\
\text { percent }\end{array}$ \\
\hline 1 & 2.64 & 66.01 & 66.01 \\
\hline 2 & .75 & 18.72 & 84.74 \\
\hline 3 & .44 & 11.05 & 95.78 \\
\hline 4 & .17 & 4.22 & 100 \\
\hline
\end{tabular}

Taking a cue from the above statistical data following rules has been proposed.

1. If $\mathrm{RSI}<60$ and MFI less than 40 then retracement is $38.2 \%$

2. If $\mathrm{RSI}<60$ and MFI greater than 40 then retracement is either $78 \%$ or $100 \%$ or more.

3. If RSI $>=60$ and MFI between 60 to 81 then retracement is either $50 \%$ or $61.8 \%$.

4. If $\mathrm{RSI}>=60$ and MFI $>81$ then retracement is $38.2 \%$.

\section{DATA ANALYSIS}

In this paper, falling wave the wave 2 retracement has been analyzed when wave 1 was the rising wave. Various test cases of NIFTY were tested on the proposed model as given in the table 3 . 
Table3 . Experimental data

\begin{tabular}{|c|c|c|c|c|}
\hline Test case Id. & RSI & MFI & $\begin{array}{l}\text { Proposed } \\
\text { Retracement }\end{array}$ & Actual retracement \\
\hline 1. & 55 & 64 & $78 \%$ or $100 \%$ & $78 \%$ \\
\hline 2. & 51 & 49 & $78 \%$ or $100 \%$ & $100 \%$ \\
\hline 3. & 59 & 63 & $78 \%$ or $100 \%$ & $100 \%$ \\
\hline$* 4$ & 61 & 84 & $38.2 \%$ & $61.8 \%$ \\
\hline 5. & 81 & 82 & $38.2 \%$ & $38.2 \%$ \\
\hline$* 6$. & 44 & 69 & $78 \%$ or $100 \%$ & $61.8 \%$ \\
\hline 7 & 40 & 56 & $78 \%$ or $100 \%$ & $78 \%$ \\
\hline 8 & 54 & 58 & $78 \%$ or $100 \%$ & $100 \%$ \\
\hline$* 9$. & 50 & 62 & $78 \%$ or $100 \%$ & $38.2 \%$ \\
\hline 10. & 55 & 51 & $78 \%$ or $100 \%$ & $100 \%$ \\
\hline$* 11$. & 72 & 81 & $50 \%$ or $61.8 \%$ & $78 \%$ \\
\hline 12. & 56 & 59 & $78 \%$ or $100 \%$ & $100 \%$ \\
\hline 13. & 75 & 82 & $38.2 \%$ & $38.2 \%$ \\
\hline 17. & 75 & 72 & $50 \%$ or $61.8 \%$ & $61.8 \%$ \\
\hline 15. & 64 & 61 & $50 \%$ or $61.8 \%$ & $61.8 \%$ \\
\hline$* 16$. & 76 & 83 & $38.2 \%$ & $50 \%$ \\
\hline 17. & 71 & 75 & $50 \%$ or $61.8 \%$ & $50 \%$ \\
\hline$* 18$. & 69 & 81 & $50 \%$ or $61.8 \%$ & $78 \%$ \\
\hline 19. & 62 & 76 & $50 \%$ or $61.8 \%$ & $61.8 \%$ \\
\hline 20. & 36 & 48 & $78 \%$ or $100 \%$ & $100 \%$ \\
\hline 21. & 86 & 79 & $50 \%$ or $61.8 \%$ & $50 \%$ \\
\hline 22. & 77 & 74 & $50 \%$ or $61.8 \%$ & $61.8 \%$ \\
\hline 23. & 55 & 48 & $78 \%$ or $100 \%$ & $78 \%$ \\
\hline 24. & 71 & 69 & $50 \%$ or $61.8 \%$ & $61.8 \%$ \\
\hline 25 & 50 & 54 & $78 \%$ or $100 \%$ & $100 \%$ \\
\hline 26. & 54 & 64 & $78 \%$ or $100 \%$ & $100 \%$ \\
\hline
\end{tabular}


Table 4. Result.

\begin{tabular}{|l|l|l|}
\hline Sr. no. & Percentage of proper classification & Percentage of misclassification \\
\hline 1. & 77 & 23. \\
\hline
\end{tabular}



Fig1. Percentage of proper classified and misclassified test cases

It was observed that about seventy seven percent of instances were properly classified by the proposed model.

\section{CONCLUSION}

Financial sector market produces huge data, Investors generally fails to predict the bottom or summit of a trend. Guessing rarely fits the trend and investors have to face the money loss. In this proposed model, only the falling wave, the wave 2 is analyzed with respect to the retracement of rising wave the wave 1 . It has been observed in this study that retracement of wave 2 with respect to wave 1 can be predicted more precisely by the proposed model. Proposed model rules are derived by the confluence of RSI and MFI , since RSI has higher Eigen values in factor analysis so RSI has been given more importance in the proposed model in formation of rules.

\section{REFERENCES}

[1] P.C Biswal ,2009,Discrete mathematics and graph theory, 2nd edition, EEE, PHI learning private limited.

[2] Darren George, Paul Mallery,2009,SPSS for windows step by step a simple guide and reference 15.0 update, 8th edition, Pearson.

[3] I.T. Jollif, Principal Component Analysis, $2^{\text {nd }}$ edition Springer, Springer series in statistics.

[4] K. Senthamarai Kannan, P. Sailapathi Sekar, M.Mohamed Sathik and P. Arumugam, march 1719,2010, Financial Stock Market Forecast using Data Mining Techniques, proceedings of the international multi conference of Engineers and computer scientists ,volume 1, Hongkong.

[5] N.K Liu ,K.K Lee,1997,An intelligent business advisor system for stock investment, expert systems Vol.14 number 3,page 129-139.

[6] Werner F.M,De Bondt, Richard Thaler, 1985,Does the stock market over react ?,The Journal of finance, Volume XL. Number 3, pp 793-805.
[7] Md. Rafiul Hassan and Baikunth Nath, 2005,StockMarket Forecasting Using Hidden Markov Model:A New Approach, Proceedings of the 5th International Conference on Intelligent Systems Design and Applications

[8] N.G Mankiw,David Romer, M.D. Shapiro,1985,An unbiased reexamination of stock market volatility, The journal of finance, Volume. XL, Number 3.

[9] T.G Anderson, T Bollerslev, F. X. Diebold, 2007,Roughing it up: including jump components in the measurement, Modeling and forecasting of return volatility, The review of economics and statistics, pp 701-720.

[10] National stock exchange of India, 2013,NCFM,Technical analysis module.

[11] Dongsong Zhang and Lina Zhou,2004, Discovering Golden Nuggets: Data Mining in Financial application, IEEE Transactions on systems , man and cybernetics part C:Application and reviews, vol 34,no 4.

[12] A. N. Refenes, A. D. Zapranis, and Y. Bentz, 1993,"Modeling stock returns with neural networks," presented at the Workshop on Neural Network Applications and Tools, London, U.K.

[13] J. Roman and A. Jameel,19966,Backpropagation and recurrent neural networks in financial analysis of multiple stock market returns, presented at the 29th HICSS, Wailea, HI.

[14] S. Walczak, 1999,Gaining competitive advantage for trading in emerging capital markets with neural networks, J. Manag. Inform. Syst., vol. 16, pp. 177-192. 